\title{
Numerical study of crystalline plasticity: measurements of the heterogeneities due to grain boundaries under small strains
}

Etude numérique de la plasticité cristalline : mesures des hétérogénéités dues aux joints de grains en petits déformations

BARBE $F .^{1,2}$, FOREST S. ${ }^{1}$, QUILICI S. ${ }^{1}$, CAILLETAUD G. $^{1}$

(1) Centre des Matériaux P.M. Fourt - UMR 7633

Ecole des Mines de Paris, 91000 EVRY, France

(2) Laboratoire de Mécanique de Rouen - UMR 6138

INSA de Rouen, 76801 SAINT ETIENNE DU ROUVRAY, France

A finite element 3D polycrystalline aggregate made of 216 grains is subjected to a mean axial deformation up to $1 \%$. The heterogeneities inside the polycrystal are due to the crystallographic orientations of the grains -this appears at the scale of the mean responses per grain- and to the local interaction between neighboring grains -this appears at the intragranular scale. The intragranular heterogeneity is measured as a function of the distance to the grain boundary, inside a single grain and for all the points of the microstructure. It is characterised by dispersions of the local responses due to grain boundaries. These measurements correspond to dispersions -variances-; the statistical errors have also been determined.

\section{Introduction}

Among all the methods developed to characterise the local behavior in polycrystalline materials, the experimental determination of the fields inside grains requires to cutoff slices of the material to enable three-dimensional measurements. That is why such measurements are often made on multicrystals with one or two grains along the shortest direction of the piece of material. Numerical representation of the local behavior inside an aggregate is possible, through homogenisation or finite elements computations [Delaire et al., 2000, Raabe et al., 2001, Lebensohn, 2001]. The heterogeneities displayed with these approaches have been confirmed by comparison to experimental measurements. From the numerous studies concerning the intragranular description of the mechanisms of deformation we know that the behavior inside a grain is governed by dislocation arrangements that may cause greatly varying responses from point to point. Dislocation cells and walls form intragranular structures with sizes/spacing depending upon the distance to a grain boundary, a so-called geometrically necessary boundary [Ashby, 1970] or even a free surface [Horstemeyer et al., 2001]. Such features have been introduced in many non-local modelings with more or less accordance to physics: strain gradient plasticity [Shu and Fleck, 1999, Acharya and Beaudoin, 2000], Cosserat plasticity [Forest et al., 2000], explicit construction of dislocation structures [Ortiz et al., 2000]...As well as experimental characterisations, these models, as long as they intend to represent intragranular heterogeneities, are restricted to structures containing few crystals.

With the use of FE parallel computations and with a classical approach to crystalline plasticity under small strains, it is possible to describe the intragranular heterogeneity in a model of three-dimensional polycrystals [Quilici and Cailletaud, 1999, Barbe et al., 2001a, Barbe et al., 2001b]. The results thus deal with a huge amount of points inside each grain - largely enough to observe variations of gradients inside grains - where the interactions / slip patterns are three-dimensional. This is a fundamental requirement to justify performing statistical considerations: the number of individuals - points - is large enough. Another requirement is that the medium be isotropic so that the analysis be valid in any direction of space. This condition is respected as it has been checked in previous works with similar number of grains and the same material [Barbe et al., 2001a, Barbe, 2000]. Consequently, all the points can be regarded as equivalent in any direction of space. In these works, we have also checked that the loading boundary conditions of the structure had negligible effect on the polycrystal effective response and on the mean response per grain. Only a small layer of one-grain-depth is affected by the boundary conditions (for a same tensile test, homogeneous strain boundary conditions were used, as well as mixed boundary conditions where the minimum necessary displacements were imposed). There thus might not be any distinction to make between points having different positions toward the boundary of the structure - at least, once all the points are mixed together through averaging operations, the slight effect due to structure boundaries vanishes-. Furthermore, we can choose not to make any distinction between points of different crystallographic orientation and classify the points regarding their minimum distance to a grain boundary. This distance thus becomes the only parameter considered for the analysis of stress/strain 
heterogeneities.

In the following, tools for the modeling of the behavior of polycrystalline aggregates and an example of computation (section 2) are briefly presented. Section 3 deals with the measurements taken from a simulated 216-grain-polycrystal subjected to mean tensile loading: first the definitions of the measurements are given and then the resulting measurements are provided for the case of our polycrystal. They consist in (i) the mean response in sets/classes of points being at a same distance to a grain boundary, (ii) the dispersion in these sets of points and (iii) the estimated error associated to the number of representative points in each set. Results are then discussed in section 3.2.

\section{Polycrystal modeling}

\subsection{Single Crystal}

It is assumed that slip is the predominant deformation mechanism and that Schmid's law is valid. The resolved shear stress can then be used as a critical variable to evaluate the inelastic flow. A viscoplastic framework is chosen, in order to avoid the problems related to the determination of the active slip systems in plastic models. A threshold is introduced both in positive and negative directions on each slip system : twelve octahedral slip systems will be used for FCC materials. Two variables are defined for each slip system $s, r^{\mathrm{s}}$ and $x^{\mathrm{s}}$, corresponding respectively to isotropic hardening (expansion of the elastic domain), and kinematic hardening (translation of the elastic domain [Chaboche, 1989]). A system will be active provided its resolved shear stress $\tau^{\mathrm{s}}$ is greater than $x^{\mathrm{s}}+r^{\mathrm{s}}$ or less than $x^{\mathrm{s}}-r^{\mathrm{s}}$ and the slip rate will be known as long as stress and the hardening variables are known. The state variables used to define the evolution of $r^{\mathrm{s}}$ and $x^{\mathrm{s}}$ are the accumulated slip $v^{\mathrm{s}}$ for isotropic hardening and the variable $\alpha^{\mathrm{s}}$ for kinematic hardening. Knowing the stress tensor $\sigma^{\mathrm{g}}$ applied to the grain $\mathrm{g}$, the resolved shear stress for system $s$ can be classically written according to (1), $\vec{n}^{\mathrm{s}}$ and $\vec{m}^{\mathrm{s}}$ being respectively, for the system $s$, the normal to the slip plane and the slip direction in this plane. The hardening variables $x^{\mathrm{s}}$ and $r^{\mathrm{s}}$ can then be expressed as a function of $\alpha^{\mathrm{s}}$ and $v^{\mathrm{s}}$ following (2), their current values allowing us to compute the viscoplastic slip rate $\dot{\gamma}^{\mathrm{s}}$, the viscoplastic strain rate tensor ${\underset{\sim}{\dot{\varepsilon}}}^{\mathrm{g}}(3)$, and the hardening rules ((2.1) and (5)). The present formulation gives a saturation of the hardening in both monotonic and cyclic loading, and takes into account the interactions between the slip systems, through matrix $h_{r s}$, as in [Kocks and Brown, 1966]. Nine material-dependent coefficients are involved in the model $\left(E, \nu, K, n, c, d, R_{0}, Q, b\right)$.

$$
\begin{gathered}
\tau^{\mathrm{s}}=\sigma_{\sim}^{\mathrm{g}}:{\underset{\sim}{m}}^{\mathrm{s}}=\frac{1}{2}{\underset{\sim}{\mathrm{g}}}^{\mathrm{g}}:\left(\vec{n}^{\mathrm{s}} \otimes \vec{m}^{\mathrm{s}}+\vec{m}^{\mathrm{s}} \otimes \vec{n}^{\mathrm{s}}\right) \\
x^{\mathrm{s}}=c \alpha^{\mathrm{s}} \quad ; \quad r^{\mathrm{s}}=R_{0}+Q \sum_{r} h_{r s}\left\{1-e^{-b v^{\mathrm{r}}}\right\} \\
\dot{\gamma}^{\mathrm{s}}=\dot{v}^{\mathrm{s}} \operatorname{sign}\left(\tau^{\mathrm{s}}-x^{\mathrm{s}}\right) ; \quad \dot{\dot{\varepsilon}}^{\mathrm{g}}=\sum_{s}{\underset{\sim}{\mathrm{m}}}^{\mathrm{s}} \dot{\gamma}^{\mathrm{s}} \\
\dot{v}^{\mathrm{s}}=\left\langle\frac{\left|\tau^{\mathrm{s}}-x^{\mathrm{s}}\right|-r^{\mathrm{s}}}{K}\right\rangle^{n}
\end{gathered}
$$

$$
\begin{array}{r}
\text { with }\langle x\rangle=\operatorname{Max}(x, 0) \quad \text { and } \quad v^{\mathrm{s}}\left(t=t_{0}\right)=0 \\
\dot{\alpha}^{\mathrm{s}}=\dot{\gamma}^{\mathrm{s}}-d \alpha^{\mathrm{s}} \dot{v}^{\mathrm{s}} \quad \text { with } \quad \alpha^{\mathrm{s}}\left(t=t_{0}\right)=0
\end{array}
$$

Such a formulation [Cailletaud, 1987, Méric et al., 1991] is an extension of the classical crystallographic approach for single crystal modeling in plasticity or in viscoplasticity (see for instance [Taylor, 1938, Mandel, 1965, Asaro, 1983]). It has been extensively used for single crystal modeling including Finite Element simulations [Méric and Cailletaud, 1991, Besson et al., 1998]. The material coefficients correspond to INCO600:

- isotropic elastic behavior: $E=196000 M P a, \quad \nu=0.3$

- viscous effect in (2.1) and (5): $K=10 M P a . s^{1 / n}, \quad n=25$

- kinematic hardening in (2): $\quad c=1600 M P a, \quad d=40$

- isotropic hardening in (2) and (3): $R_{0}=111 M P a, Q=$ $35 M P a, \quad b=7$

\subsection{Computation and as-given results}

The constitutive laws for single crystals are applied to each integration point of a FE structure representing a polycrystal. Each grain is made of a set of Gauss points that represents a Voronoï polyhedron (see fig.1a for illustration and [Barbe et al., 2001a] for the details about Voronoï polyedra generation - contribution due to Decker and Jeulin, LMM-ENSMP-). The dependence of our results at different scales on the mesh refinement has been determined in [Barbe et al., 2001c]. At least $4 \times 4 \times 4$ 20-nodefull-integration-elements per grain must be used to expect a correct result at the intragranular scale. The results produced in the following analysis deal with a structure made of $36 \times 36 \times 36$ elements (1259712 integration points). For such a big structure, we have resorted to parallel computing on 32 processors of the Linux PC Cluster of Centre des Matériaux (Ecole des Mines de Paris): the structure was divided into 32 sub-domains computed separately and balanced through the FETI method at each increment of the load [Feyel, 1998, Quilici and Cailletaud, 1999]. As an example of the results obtained from such computations, the contour of the amount of plastic slip after $1.5 \%$ axial strain on a 200-grain-polycrystal is shown on fig. $1 \mathrm{~b}$. It displays the large variation in the local values at the intragranular scale. But our purpose here is to quantify those variations; it is referred to [Barbe et al., 2001b, Barbe, 2000] for a detailed analysis of as-given results. In the following, the analysis focuses on results of an axial tensile test to $1 \%$ total strain performed on a 216-grain-polycrystal.

\section{Measurements of hetero- geneities}

\subsection{Definitions}

Each point of the structure is an individual which has a proper set of values, one for each mechanical variable $\left(\varepsilon_{e q}\right.$ and $\sigma_{e q}$ will be treated in the following). Collecting all the points of a grain or of the whole structure at a same distance to the grain boundary ensemble, we build classes of individuals, one class per mechanical variable, that we consider to be discrete random variables. Then we can 
try to find an estimator for each variable, and the related dispersion - square root of the variance - and error. The most direct estimator suited to our case is the esperance, i.e. the normal averaging over all the points at a same distance to a grain boundary. With the assumption that this estimator is correct, we compute the relative dispersion (7) around the estimation and the relative error (8) that is made by choosing the average as estimator.

Let us first call $X_{d_{i}}$, the discrete random variable defined by the set of responses, either $\varepsilon_{e q}$ or $\sigma_{e q}$, at a given distance $d_{i}$ to the grain boundary ensemble: $X_{d_{i}}=\left\{x_{k}\right\}_{\left(k=1, N\left(d_{i}\right)\right)}$ where $N\left(d_{i}\right)$ is the number of points at the distance $d_{i} \pm$ $\left(d_{i}-d_{i-1}\right) / 2$.

The absolute dispersion for this variable $X_{d_{i}}$ is the square root of the variance:

$$
D_{a b s}\left(d_{i}\right)=\sqrt{\frac{\sum_{k=1}^{N\left(d_{i}\right)}\left(x_{k}-<X_{d_{i}}>\right)^{2}}{N\left(d_{i}\right)}}
$$

where $<X_{d_{i}}>=\sum_{k=1}^{N\left(d_{i}\right)} x_{k} / N\left(d_{i}\right)$ is the mean value of $X_{d_{i}}$.

The relative dispersion is the dispersion normalized by the mean value $<X_{d_{i}}>$ :

$$
D_{r e l}\left(d_{i}\right)=\frac{D_{a b s}\left(d_{i}\right)}{<X_{d_{i}}>}
$$

Relative error associated to the number of representatives:

$$
E_{r e l}=\frac{D_{a b s}}{\left\langle x_{k}>_{d_{i}}\right.} \cdot \frac{1}{\sqrt{N\left(d_{i}\right)}}
$$

The measurements are made for all the points of a single grain and then considering the points of the structure alltogether, without any care on their grain belonging. In the second case, there are points of a same class (same distance to a grain boundary) that have different distances to their respective grain centers. In other words, the maximum distance to a grain boundary varies from grain to grain, which is illustrated on fig. $2 \mathrm{a}$ that gives the distribution among grains of this maximum distance to a grain boundary. But doing so, one makes estimations on larger sets of individuals than if a single grain were treated. The total number of points of the structure in each class of distance to a grain boundary is given on fig. $2 \mathrm{~b}$. As for other graphs featuring the distance to a grain boundary, the measurements are performed in intervals of distances to the grain boundary having two different sizes: 0.01 (the set of distances then corresponds nearly to the set of every possible discrete distance) and 0.1 which is less than one tenth of the minimum distance to a grain boundary. It is thus seen that measurements for each possible discrete distance shall be considered carefully if the distance to the grain boundary exceeds 2.5 whereas measurements in intervals sized 0.1 do not suffer from lack of representatives until the distance 3 is attained.

The distance from an integration point to the grain boundary is defined as the distance between the point concerned and the closest integration point located along the grain boundary inside the same grain. For this reason, for any integration point of the structure, the minimum distance to the grain boundary is necessarily 0 .

\subsection{Results and observations}

\section{In a single grain}

First, von Mises equivalent stress and strain inside a single grain are considered on fig. 3 where all the local responses are given as functions of the distance to the grain boundary, together with the normal average response per interval of distance to the grain boundary. Apart from the increasing variation of the responses as a grain boundary is approached, it is noticeable that the normal average made in intervals of distance does not vary much from grain center to grain boundary (about $10 \%$ of variation in strain and $5 \%$ in stress). If the normal average over all the points of a class is taken as the estimator, on fig. 4 , we see that there is an increasing dispersion from the grain center to the grain boundary for both stress and strain. But it is greater with strain (about $30 \%$ ) than with stress (about 5\%). The associated error (on fig.5) shall be interpreted carefully: it does not vary although an increase could be expected around the grain center where number of representatives becomes very low; the largest errors are even reached on points far from grain center. So we actually just can deduce that the dispersions measured have a statistical validity, at least for distances less than 2.5 where stress and strain obviously appear to be largely dispersed among a large number of representatives (fig.3). For longer distances, the number of representatives becomes very low and in the extreme limit, there is just a single point per class.

\section{In the whole structure}

The same plots of (i) the mean response per class of points, (ii) the dispersion and (iii) the error, determined from all the points of the structure, are given in fig.6,7,8. Fig. 6 confirms the tendency featured by the single grain analysed: there is no significant variation of the mean behavior with the distance to the grain boundary. There is just a slight $6 \%$ monotonous decrease in strain from the distance 0.5 to the distance 2.5. About stress, one can observe a tendency to increase in a monotonous manner in the interval $[0.7,2.5]$ but it is too low an increase to be a significant effect (about $1.5 \%$ increase in stress ...). Moreover, from the analysis of another polycrystal of INCO600 containing 200 grains, it has been found varying signs of the variations of stress/strain from grain centers to grain boundaries [Barbe, 2000]. These variations were also very low so there is no conclusion to draw from the slight variations observed. Nevertheless, from a detailed analysis of the mechanisms of slip (number of slip systems having a slip [resp. resolved shear stress] superior to a fixed value, number of systems having slip [resp. resolved shear stress] superior to a certain proportion of the maximum slip [resp. resolved shear stress] ...), it has been observed qualitative variations with distance to a grain boundary consistent with the results of the analysis of free surface effect: a free surface, compared to the behavior in the bulk, favors higher strains and lower stresses by allowing activated slip systems to reach a great amount of slip; by contrast, a grain boundary tends to activate more slip systems while limiting the amount of slip on these systems. The analysis can actually not be restricted to the simple consideration of stress/strain and number of activated slip systems: there could be no effect on the stress/strain while there is an effect on the mechanisms of plasticity; the analysis requires to clearly differentiate the ways a slip system can be defined to be active. So, as a conclusion to the previous observations, 
one just can say that there is no significant effect of the grain boundaries on the mean response of points at a same distance to the grain boundaries. Unlike the widespread opinion that grain boundaries act as hardening layers that reinforce the behavior of each grain and that are taken to be responsible for the hardening of a polycrystal by comparison to single crystals alone [Hirth, 1972, Mughrabi, 1983, Argon and Haasen, 1993, Fu et al., 2001], there is no hardening nor softening effect due to the grain boundaries in our results. Some of these characteristics have been observed in the experimental measurements of Raabe et al [Raabe et al., 2001] on a quasi 2D polycrystalline layer of aluminium strained at $8 \%$ : a spectrum of $-87.5 \%$ to $+87.5 \%$ deviation from the average value has been measured; some regions near grain boundaries correspond to strain localisation whereas some others do not.

The quantitative dispersion and the qualitative variation of the dispersion with the distance to a grain boundary (fig.7) are also confirmed: $30 \%$ of dispersion in strain along the grain boundaries and $17 \%$ in stress (i.e. about half the dispersion in strain). From fig. $2 \mathrm{~b}$ we know that there just remain about 60 grains (a quarter) having a maximum distance to their boundary superior to 2.5 . Those 60 grains occupy the volume fraction $90 / 216$ i.e. $42 \%$ of the whole structure. One reasonably can suppose that the behavior inside these grains might not be so particular that their responses are not significant of a 'normal' behavior, especially since they are the biggest grains, those who offer the best conditions for statistical considerations. Accordingly, one can bear on the measurements made between the distance 0 to 2.5 ; the remaining part of distances is representative of the behavior in the core of the biggest grains. So a linear variation of the dispersion of strain from $30 \%$ at the grain boundary to $20 \%$ at distance 2.5 is observed. In stress, this variation goes from $17 \%$ to $12.5 \%$, nearly linearly as well.

The error arising from the number of discretisation points confirms that one can lay on our measurements for the major part of intragranular regions, at least for distances from 0 to 2.5 : it reaches about $2 \%$ in strain and $1 \%$ in stress for a distance 2.5. Above this distance, the phenomenon expected in the single grain is present: the error remarkably increases, especially when it is measured in intervals sized 0.01 . Indeed, the individuals of a class made from all the points of the structure have a priori a more pronounced random character than the points of a class inside a single grain: the behavior in a grain may be significantly different from the one of another grain (for $1 \%$ total axial strain on this polycrystal, the mean response per grain varies from 250 to $500 \mathrm{MPa}$ in stress and from 0.06 to 0.14 in strain) whereas two points of a same grain located near the center have no reason to vary greatly around the response at the center.

\section{Conclusion}

It has been shown that, in a purely local mechanical modeling of the behavior of polycrystal, where no variation of the constitutive behavior with position towards a grain boundary or any type of interacting region is accounted for and where only the geometrical arrangement of crystals generates intragranular heterogeneities, the major effect of the grain boundaries is the large dispersion that they cause; only the anisotropy of plasticity combined to structural heterogeneity is responsible for this in our case. Grain boundaries may favor localisation in some places but no localisation appears by average: grain boundaries do not harden nor soften the behavior in their surroundings.

\section{References}

[Acharya and Beaudoin, 2000] Acharya, A. and Beaudoin, A. J. (2000). Grain size effect in viscoplastic polycrystals at moderate strains. J Mech Phys Sol, 48(10):2213-2230.

[Argon and Haasen, 1993] Argon, A. S. and Haasen, P. (1993). A new mechanism of work-hardening in the late stages of large strain plastic flow in F.C.C. and diamond cubic crystals. Acta metall mater, 41(11):3289-3306.

[Asaro, 1983] Asaro, R. J. (1983). Crystal Plasticity. J. Appl. Mech., 50:921-934.

[Ashby, 1970] Ashby, M. (1970). The Deformation of Plastically Non-homogeneous Materials. Phil Mag, 21:399-424.

[Barbe, 2000] Barbe, F. (2000). Etude numérique de la plasticité d'agrégats polycristallins. $\mathrm{PhD}$ thesis, Ecole Nationale Supérieure des Mines de Paris.

[Barbe et al., 2001a] Barbe, F., Decker, L., Jeulin, D., and Cailletaud, G. (2001a). Intergranular and intragranular behavior of polycrystalline aggregates. Part 1: F.E. model. Int J Plasticity, 17:513-536.

[Barbe et al., 2001b] Barbe, F., Forest, S., and Cailletaud, G. (2001b). Intergranular and intragranular behavior of polycrystalline aggregates. Part 2: results. Int J Plasticity, $17: 537-563$.

[Barbe et al., 2001c] Barbe, F., Forest, S., and Cailletaud, G. (2001c). Polycrystalline plasticity under small strains. Microstructure refinment. In E. Bouchaud et al, editor, NATO Proceedings, Physical Aspects of Fracture. Kluwer Publisher.

[Besson et al., 1998] Besson, J., Leriche, R., Foerch, R., and Cailletaud, G. (1998). Object-Oriented Programming Applied to the Finite Element Method. Part II. Application to Material Behaviors. Revue Européenne des Éléments Finis, 7(5):567-588.

[Cailletaud, 1987] Cailletaud, G. (1987). Une approche micromécanique phénoménologique du comportement inélastique des métaux. $\mathrm{PhD}$ thesis, Université Pierre et Marie Curie, Paris 6.

[Chaboche, 1989] Chaboche, J.-L. (1989). Constitutive Equations for Cyclic Plasticity and Cyclic Viscoplasticity. Int J Plasticity, 5:247-302.

[Delaire et al., 2000] Delaire, F., Raphanel, J., and Rey, C. (2000). Plastic heterogeneities of a copper multicrystal deformed in uniaxial tension: experimental study and finite element simulations. Acta mater, 48:1075-1087.

[Feyel, 1998] Feyel, F. (1998). Application du calcul parallèle aux modèles à grand nombre de variables internes. $\mathrm{PhD}$ thesis, Ecole Nationale Supérieure des Mines de Paris.

[Forest et al., 2000] Forest, S., Barbe, F., and Cailletaud, G. (2000). Cosserat modelling of size effects in crystals. Int $J$ Sol Struct, 37(46-47):7105-7126. 
[Fu et al., 2001] Fu, H. H., Benson, D. J., and Meyers, M. A. (2001). Analytical and computational description of effect of grain size on yield stress of metals. Acta mater, 49:25672582.

[Hirth, 1972] Hirth, J. (1972). The influence of Grain Boundaries on Mechanical Properties. Metall Trans, 3:3047-3067.

[Horstemeyer et al., 2001] Horstemeyer, M. F., Baskes, M. I., and Plimpton, S. J. (2001). Length scale and time scale effects on the plastic flow of fcc metals. Acta mater, 49:4363-4374.

[Kocks and Brown, 1966] Kocks, U. and Brown, T. (1966). Latent Hardening in Aluminium. Acta Metall, 14:87-98.

[Lebensohn, 2001] Lebensohn, R. A. (2001). N-site modeling of a $3 \mathrm{D}$ viscoplastic polycrystal using Fast Fourier Transform. Acta mater, 49:2723-2737.

[Mandel, 1965] Mandel, J. (1965). Une généralisation de la théorie de la plasticité de W.T. Koiter. Int $J$ Sol Struct, 1:273-295.

[Méric and Cailletaud, 1991] Méric, L. and Cailletaud, G. (1991). Single Crystal Modeling for Structural Calculations. Part 2: Finite Element Implementation. $J$ Engng Mat Technol, 113:171-182.

[Méric et al., 1991] Méric, L., Poubanne, P., and Cailletaud, G. (1991). Single Crystal Modeling for Structural Calculations. Part 1: Model Presentation. J Engng Mat Technol, 113:162-170.

[Mughrabi, 1983] Mughrabi, H. (1983). . Acta Metall, $31: 1367$.
[Ortiz et al., 2000] Ortiz, M., Repetto, E. A., and Stainier L. (2000). A theory of subgrain dislocation structures. $J$ Mech Phys Sol, 48:2077-2114.

[Quilici and Cailletaud, 1999] Quilici, S. and Cailletaud, G. (1999). F.E. simulation of macro-, meso- and micro- scales in polycrystalline plasticity. Comput Mat Sc, 16(1-4):383390.

[Raabe et al., 2001] Raabe, D., Sachtleber, M., Zhao, Z., Roters, F., and Zaefferer, S. (2001). Micromechanical and macromechanical effects in grain scale polycrystal plasticity - experimentation and simulation. Acta mater, 49:3433-3441.

[Shu and Fleck, 1999] Shu, J. and Fleck, N. (1999). Strain gradient crystal plasticity: size-dependent deformation of bicrystals. J Mech Phys Sol, 47:297-324.

[Taylor, 1938] Taylor, G. (1938). Plastic Strain in Metals. J. Inst. Metals, 62:307-324.

Corresponding author: Fabrice BARBE, Laboratoire de Mécanique de Rouen, INSA de Rouen BP 08, Avenue de l'Université 76801 Saint Etienne du Rouvray, cedex - France mail: fabrice.barbe@insa-rouen.fr tel: 33232959760 , fax: 33232959710 

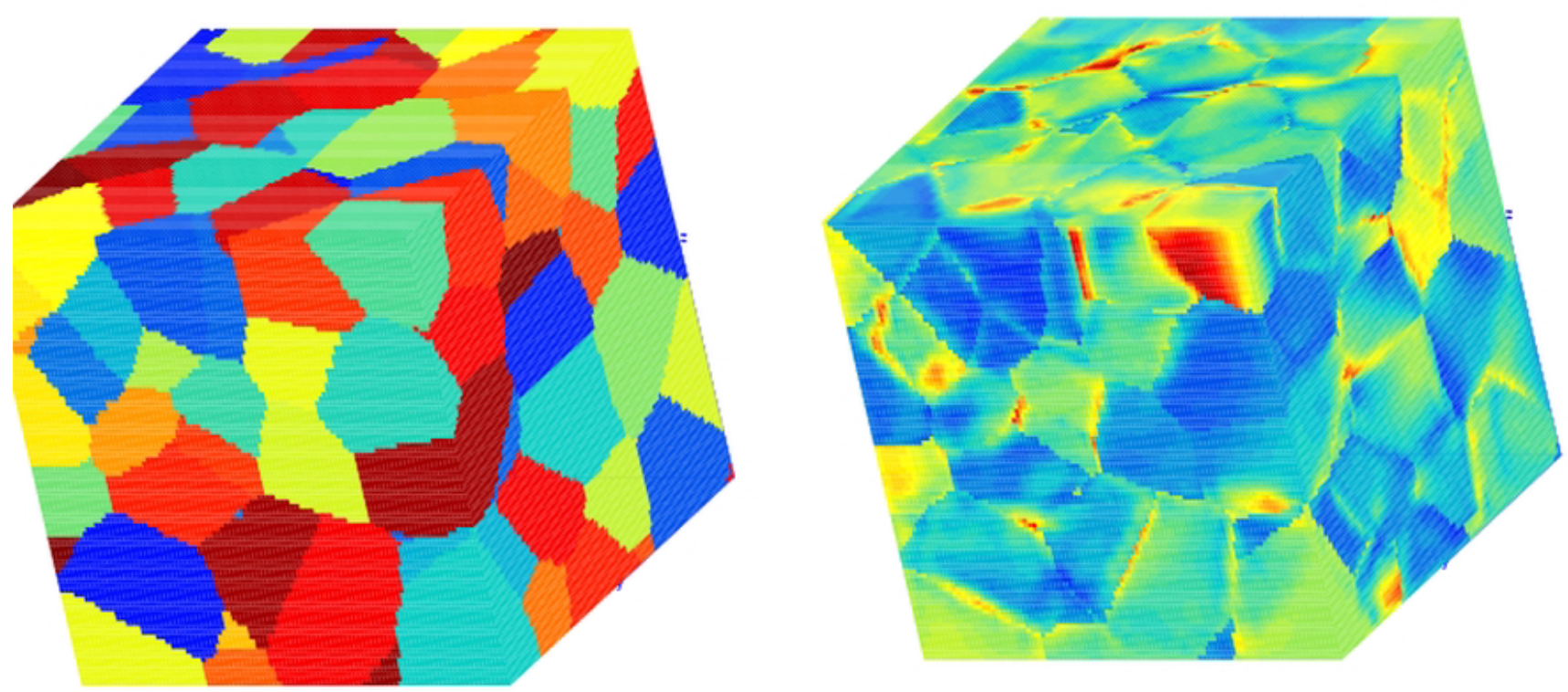

Figure 1: (Left) Polycrystalline aggregate made of 200 grains represented by $32^{3}$ full integration elements. (Right) Amount of plastic slip on the contour of the polycrystal -INCO600- loaded at $1.5 \%$ axial strain with homogeneous boundary conditions.

(Gauche) Agrégat polycristallin à 200 grains représenté par $32^{3}$ éléments briques à intégration complète. (Droite) Quantité de glissement plastique sur le contour du polycristal -INCO600- sous chargement en déformation axiale à 1, 5\%, avec des conditions homogènes sur le contour.

$[!]$ 

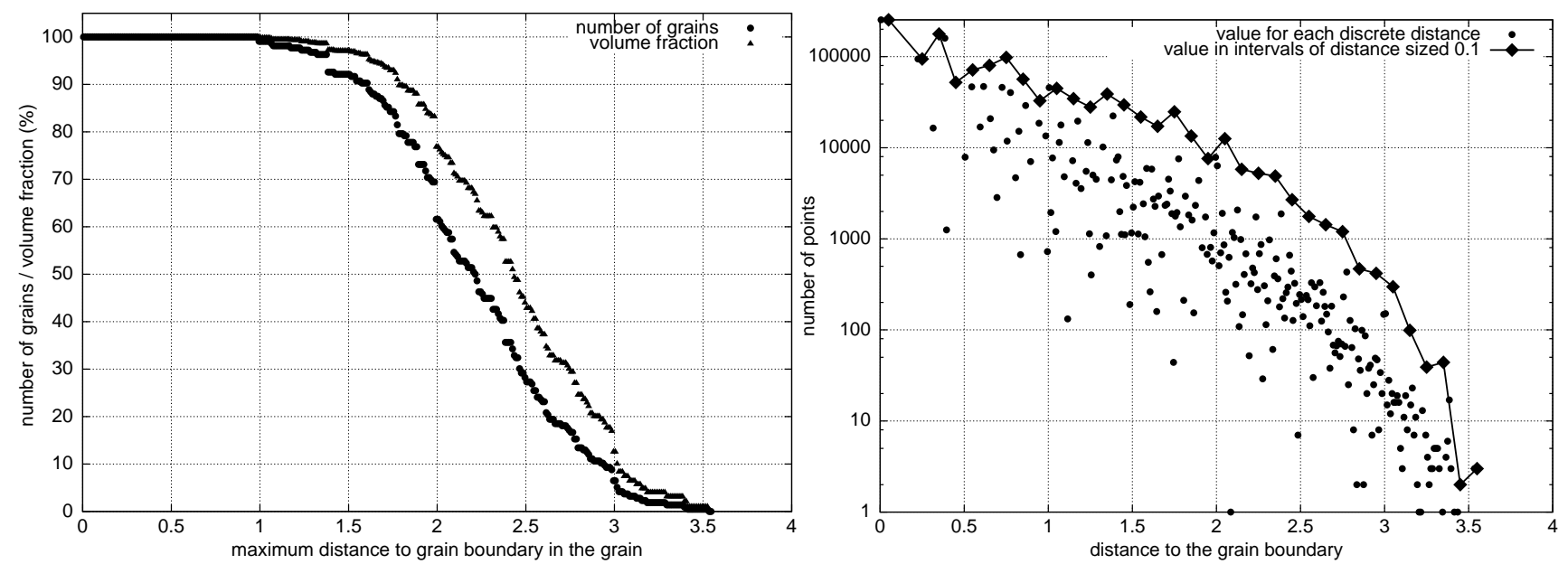

Figure 2: (Left) Distribution of the maximum distance to the grain boundary of each grain and the corresponding volume fraction: for example, $61 \%$ of the grains have a maximum distance equal to 2.0 and they represent a volume fraction of about $76 \%$. (Right) Number of points in each interval of distance to a grain boundary sized 0.01 ( $0.3 \%$ of the maximum distance) or $\mathbf{0 . 1}$ ( $3 \%$ of the maximum distance), for the whole structure

(Gauche) Distribution de la distance maximum au joint de grain de chaque grain et fraction volumique correspondante : par exemple, $61 \%$ des grains ont une distance maximum égale à 2,0 et ils représentent une fraction volumique de $76 \%$. (Droite) Nombre de points dans chaque intervalle de distance au joint de grain de taille 0,01 ( $0.3 \%$ de la distance maximum) ou 0,1 ( $3 \%$ de la distance maximum), pour la structure totale 

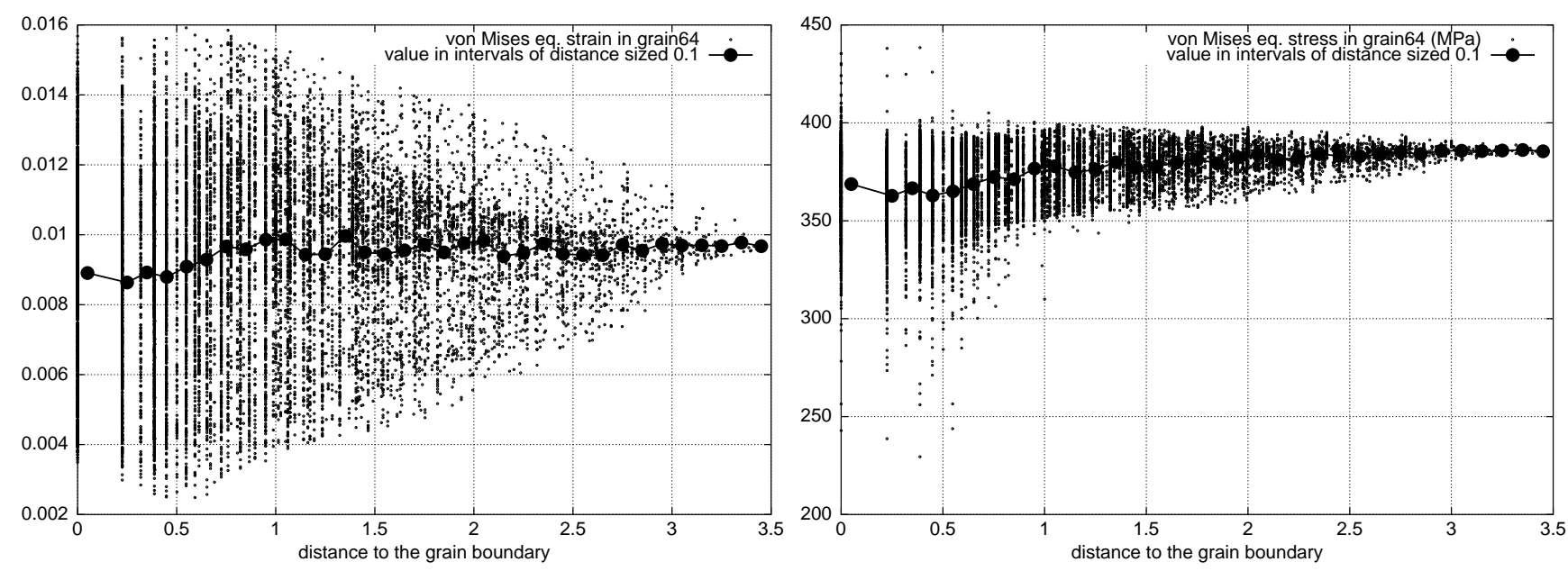

Figure 3: (Left) $\varepsilon_{e q}$ and (Right) $\sigma_{e q}(\mathbf{M P a})$ as a function of the distance to the grain boundary in a single grain

(Gauche) $\varepsilon_{e q}$ et (Droite) $\sigma_{e q}(\mathrm{MPa})$ en fonction de la distance au joint de grain dans un seul grain 

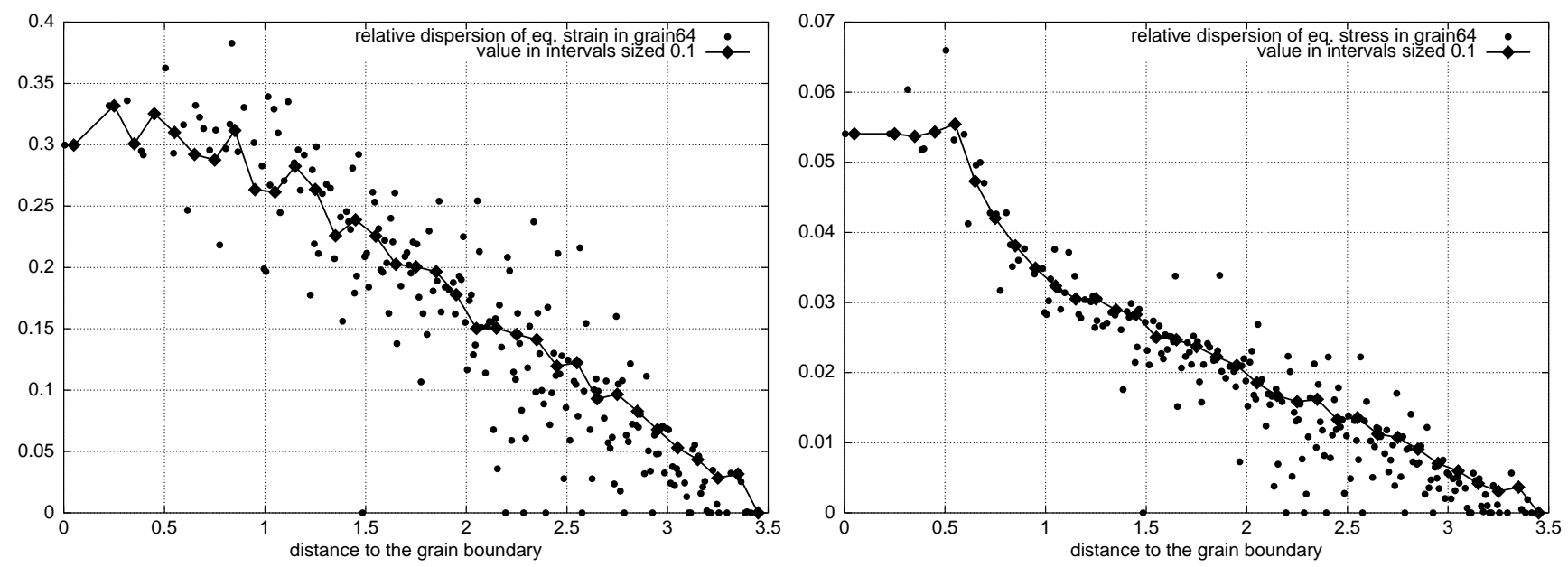

Figure 4: Relative dispersion for $\varepsilon_{e q}$ (Left) and $\sigma_{e q}$ (Right) as a function of the distance to the grain boundary in a single grain

Dispersion relative de $\varepsilon_{e q}$ (Gauche) et $\sigma_{e q}$ (Droite) en fonction de la distance au joint de grain dans un seul grain 

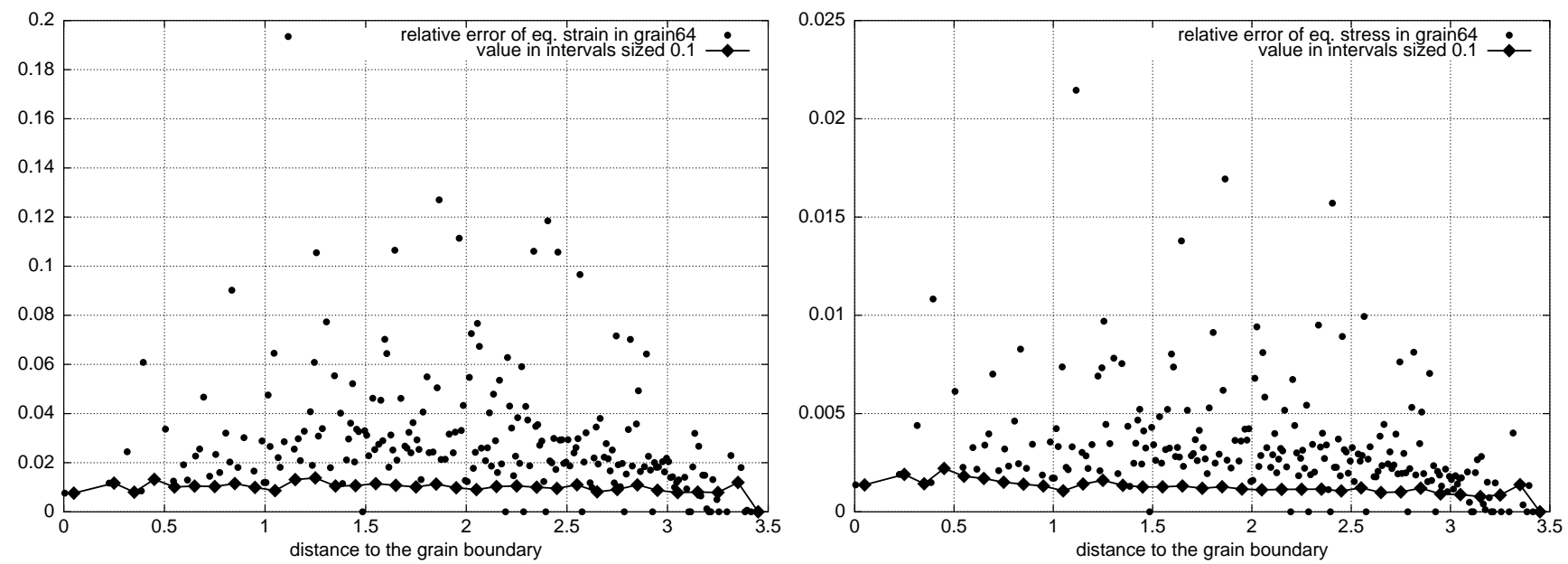

Figure 5: Relative error for $\varepsilon_{e q}$ (Left) and $\sigma_{e q}$ (Right) as a function of the distance to the grain boundary in a single grain

Erreur relative sur $\varepsilon_{e q}$ (Gauche) et $\sigma_{e q}$ (Droite) en fonction de la distance au joint de grain dans un seul grain 

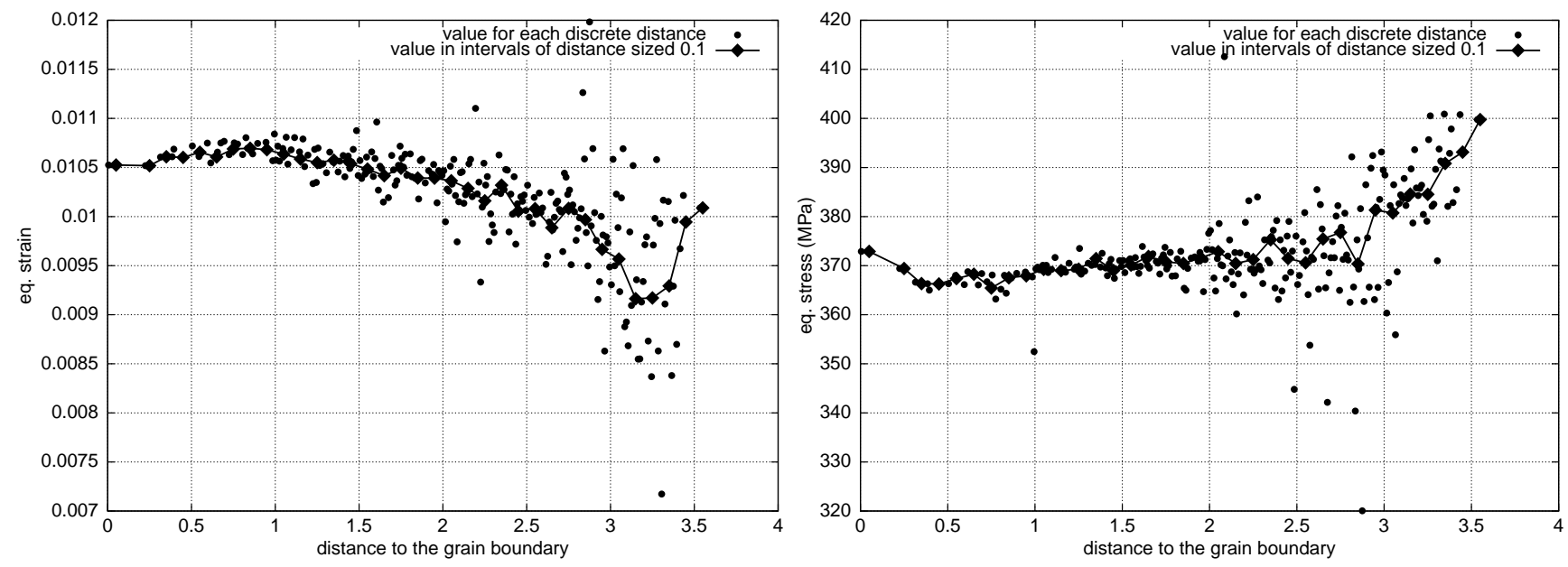

Figure 6: (Left) $\varepsilon_{e q}$ and (Right) $\sigma_{e q}$ (MPa) as a function of the distance to the grain boundaries, determined from the responses at every point of the structure

(Gauche) $\varepsilon_{e q}$ et (Droite) $\sigma_{e q}(\mathrm{MPa})$ en fonction de la distance aux joints de grains, déterminés à partir des réponses en tout point de la structure

$[!]$ 

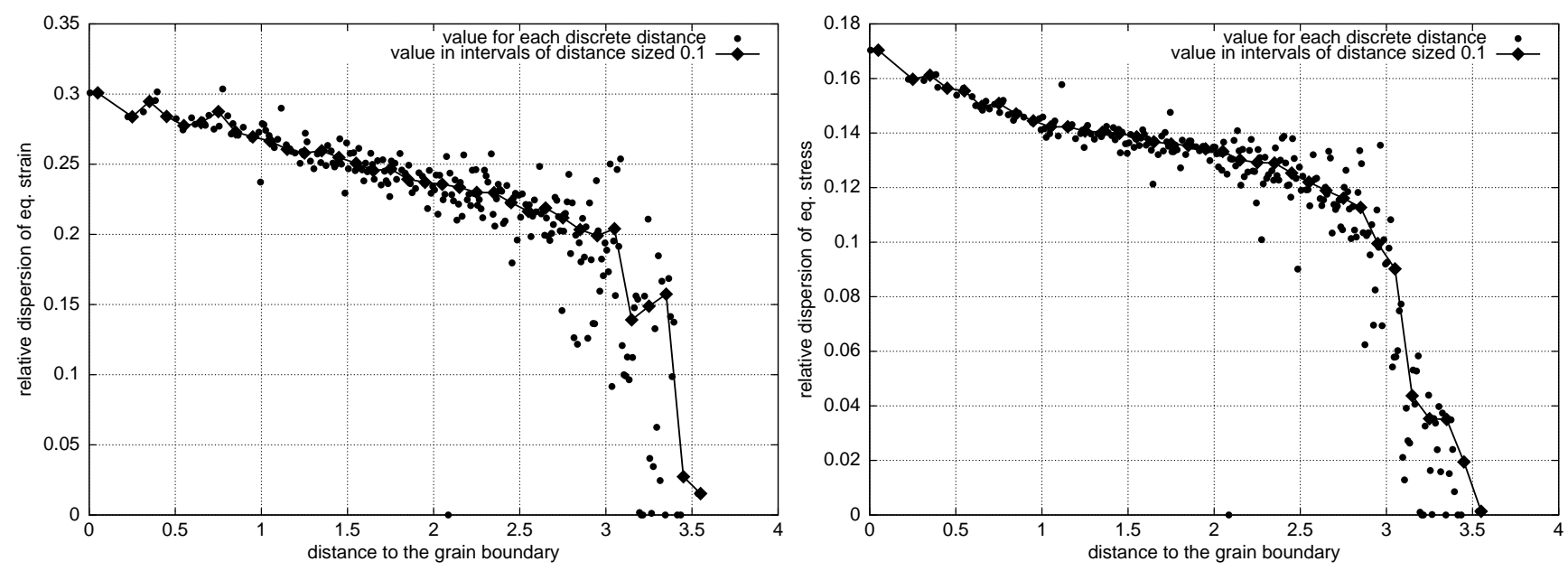

Figure 7: Relative dispersion of $\varepsilon_{e q}$ (Left) and $\sigma_{e q}$ (Right) as a function of the distance to the grain boundaries, determined from the responses at every point of the structure

Dispersion relative de $\varepsilon_{e q}$ (Gauche) et de $\sigma_{e q}$ (Droite) en fonction de la distance aux joints de grains, déterminées à partir des réponses en tout point de la structure

$[!]$ 

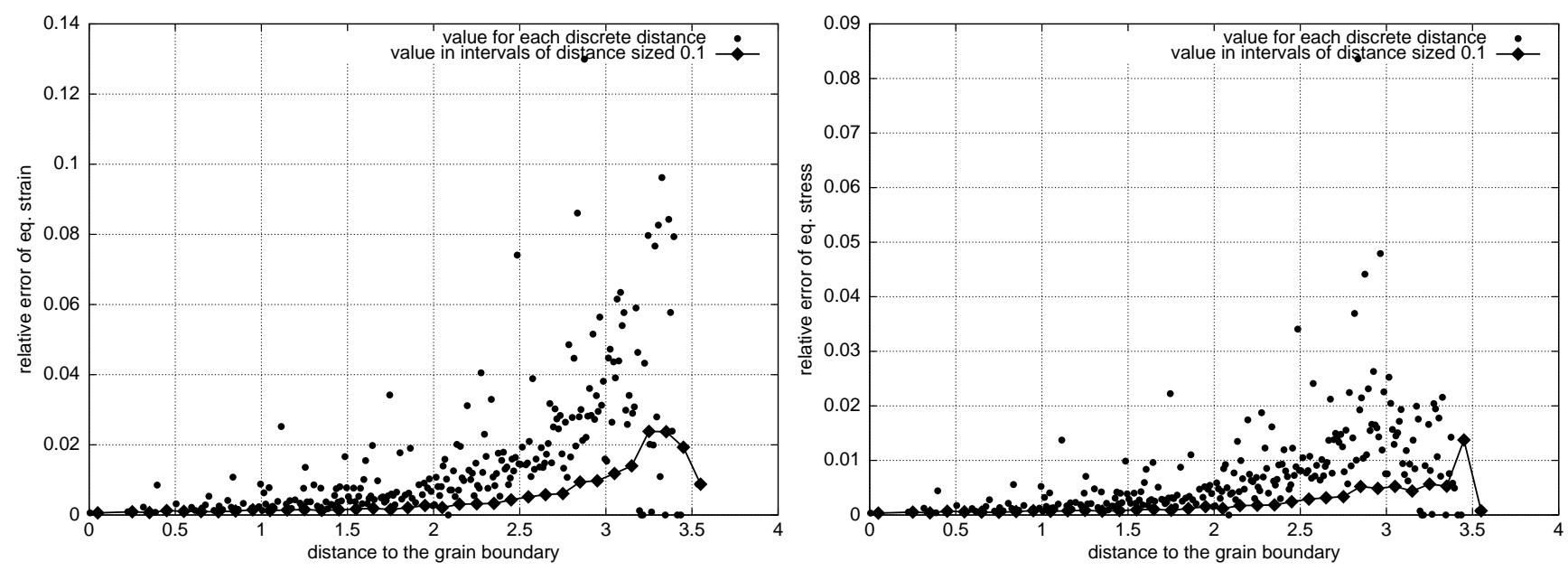

Figure 8: Relative error for $\varepsilon_{e q}$ (Left) and $\sigma_{e q}$ (Right) as a function of the distance to the grain boundaries, determined from the responses at every point of the structure

Erreur relative pour $\varepsilon_{e q}$ (Gauche) et $\sigma_{e q}$ (Droite) en fonction de la distance aux joints de grains, déterminées à partir des réponses en tout point de la structure

$[!]$ 